\title{
Entwicklung der Rechtsprechung des EuGH zum Arbeitsrecht aus der Sicht eines deutschen Arbeitsrichters
}

\author{
Hellmut WI $\beta M A N N$
}

In der Landschaft des Arbeitsrechts hat der EuGH eine Menge deutlicher Markierungspunkte gesetzt. Sie finden sich überaus reichhaltig und detailliert zu den Verboten der Diskriminierung wegen der Staatsangehörigkeit und des Geschlechts sowie zum Betriebsübergang. Auch zu einzelnen Aspekten des Arbeitszeitrechts und des Rechts der Massenentlassung hat sich der Gerichtshof mehrfach geäußert, allenfalls sporadisch dagegen zu anderen Teilen des Arbeitsrechts, fast gar nicht zum kollektiven Arbeitsrecht. Es kann nicht mein Ehrgeiz sein, die Inhalte dieser Rechtsprechung zu beschreiben. Dazu reicht die Zeit nicht, außerdem sind Persönlichkeiten unter uns, die hierzu viel eher berufen wären. Meine Aufgabe sehe ich vielmehr darin, einige Anmerkungen zur Rezeption der EuGH-Rechtsprechung im deutschen Arbeitsrecht, besonders auch in der Rechtsprechung des Bundesarbeitsgerichts, zu machen.

\section{Der EuGH als Impulsgeber im Arbeitsrecht}

Die Rechtsprechung des EuGH bildet kein geschlossenes Orientierungssystem. Der Gerichtshof könnte ein solches auch gar nicht schaffen. Das liegt nicht nur daran, dass Gerichte immer nur die Fragen beantworten können, die in Prozessen an sie herangetragen werden. Fast noch wichtiger ist im Fall des EuGH, dass das Arbeitsrecht der EG kein umfassendes, nach einer Gesamtkonzeption gestaltetes Regelwerk ist. Vielmehr besteht es aus Regelungsinseln, zwischen denen es oft kaum Beziehungen gibt. Das ist im EG tendenziell so angelegt, wenn man auch bei der Lektüre des Grünbuchs Arbeitsrecht der Kommission auf andere Gedanken kommen könnte. Ich erinnere an das Subsidiaritätsprinzip des Art. 5 EG, das auch in Art. 137 EG seinen Ausdruck findet: Danach beschränkt sich die Kompetenz der Gemeinschaft zur Setzung arbeitsrechtlichen Sekundärrechts darauf, die Tätigkeit der Mitgliedstaaten zu unterstützen und zu ergänzen; Arbeitsentgelt, Koalitionsrecht und Arbeitskampfrecht sind ganz ausgenommen. Der EG enthält also eine Überlebensgarantie für das nationale Arbeitsrecht. Überdies setzt das Gemeinschaftsrecht meist nur Mindeststandards, die vom nationalen Arbeitsrecht oft übertroffen werden, z.B. im Betriebsverfassungsrecht. Die notwendige Orientierung erfährt der Anwender des Arbeitsrechts daher nur aus dem Zusammenspiel von europäischer und innerstaatlicher Normsetzung sowie von europäischer und nationaler Judikatur. Damit ist übrigens die gelegentlich gestellte Frage, ob denn das BAG in einem im- 
mer stärker europäisch durchwirkten Arbeitsrecht künftig bedeutungslos werde, klar zu verneinen.

In diesem Rahmen verdanken wir dem EuGH allerdings kraftvolle Impulse. Denken Sie nur an den Kampf gegen die Diskriminierung wegen des Geschlechts: Er wäre bei uns ohne den Druck des Gemeinschaftsrechts, in seiner Konkretisierung durch den EuGH, sicher viel weniger effektiv geführt worden. So hat der deutsche Gesetzgeber lange geglaubt, als Sanktion im Fall der Ablehnung einer Bewerberin wegen des Geschlechts genüge ein Anspruch auf Ersatz der Kosten des Bewerbungsschreibens. Erst beharrliches Bohren auf der Grundlage von Urteilen des $\mathrm{EuGH}^{1}$ führte zu einer wirksamen Sanktionsregelung.

Oder nehmen Sie das Verbot der mittelbaren Diskriminierung: Heute ist die Erkenntnis Allgemeingut, dass eine solche Diskriminierung keine Diskriminierungsabsicht des Handelnden voraussetzt, also keinen böswillig handelnden Arbeitgeber. Die Diskriminierung ist vielmehr anhand objektiver Kriterien feststellbar, die sich auf die Auswirkungen einer Handlung oder Rechtsnorm beziehen ${ }^{2}$. Dass sich diese Erkenntnis bereits seit den späten achtziger Jahren auch in Deutschland immer mehr durchgesetzt ${ }^{3}$ und damit den Diskriminierungsverboten zu einer bis dahin unvorstellbaren Breitenwirkung verholfen hat, verdanken wir dem überzeugenden Druck der Rechtsprechung des EuGH: zunächst vorsichtig im Urteil „Jenkins“4, dann entschieden in den Urteilen „Bilka“5 und „Rinner-Kühn“66.

Diese Beispiele weisen auf ein Element, das die gesamte Rechtsprechung des EuGH durchzieht: den Grundsatz, nach dem Normen des Gemeinschaftsrechts so auszulegen sind, dass sie größtmögliche Wirksamkeit entfalten, also die Lehre vom „effet utile“7. Zwar gehört schon seit langem auch zum Auslegungskanon deutscher Richter an prominenter Stelle der Normzweck. Bei der Ermittlung und Durchsetzung dieses Zwecks wird aber nach deutscher Methodenlehre dem Wortlaut der Norm wesentlich höhere Bedeutung beigemessen, als das der EuGH hinsichtlich des Gemeinschaftsrechts tut. Er emanzipiert sich oft bemerkenswert weit vom Normtext, der in seiner Vielsprachigkeit auch wenig verlässlich ist. Der EuGH vertraut mehr auf teleologische Erwägungen.

Plastisch wird das im Urteil „Kühne \& Nagel“8: Die Richtlinie über Europäische Betriebsräte stattet nationale Arbeitnehmervertretungen mit Unterstützungsansprüchen

1 EuGH v. 10. 4. 1984, Rs. 14/83 „v. Colson u. Kamann“, AP BGB § 611 a Nr. 1; v. 8. 11. 1990, Rs. 177/88, „Dekker“, AP EWG-Vertrag Art. 119 Nr. 23.

2 Seit einigen Jahren ist das auch im Sekundärrecht der EG ausdrücklich geregelt, s. nur Art. 2 Abs. 2 Buchst. b der Richtlinie 2000/43/EG und Art. 2 Abs. 2 Buchst. b der Richtlinie 2000/78/EG.

3 Z.B. BAG v. 14. 10. 1986, AP EWG-Vertrag Art. 119 Nr. 11; v. 2. 12. 1992, AP BAT § 23 a Nr. 28.

4 EuGH v. 31. 3. 1981, Rs. 96/80, AP EWG-Vertrag Art. 119 Nr. 2.

5 EuGH v. 5. 6. 1984, Rs. 170/84, AP EWG-Vertrag Art. 119 Nr. 3.

6 EuGH v. 13. 7. 1989, Rs. 171/88, AP EWG-Vertrag Art. 119 Nr. 16.

7 Z.B. EuGH v. 29. 3. 2001, Rs. C-62/99 ,bofrost“, AP EWG-Richtlinie 94/45 Nr. 2.

8 EuGH v. 13. 1. 2004, Rs. C-440/00, AP EWG-Richtlinie 94/45 Nr. 3. 
gegen die Konzernspitze aus, damit sie für die Unternehmensgruppe die Errichtung eines Europäischen Betriebsrats ins Werk setzen können. Diese Ansprüche umfassen u. a. Informationen über Standorte und Arbeitnehmerzahlen anderer Unternehmen der betreffenden Gruppe. Sitzt allerdings die Spitze der Unternehmensgruppe in einem Drittstaat, so scheidet sie als Anspruchsschuldnerin aus. Nach der Richtlinie ist dann ein nachgeordnetes Unternehmen mit Sitz innerhalb der EG in Pflicht zu nehmen. Wie dieses Unternehmen an die geschuldeten Informationen kommt, falls die anderen Unternehmen der Gruppe nicht kooperationswillig sind, ergibt sich aus dem Wortlaut der Richtlinie nicht. Im Umgang mit nationalem Recht würde der deutsche Richter in einer solchen Situation lange darüber nachdenken, ob insoweit eine unbeabsichtigte Regelungslücke vorliegt, diese dann vielleicht bejahen, aber mit Bauchgrimmen (immerhin hat die Norm den Fall der Unternehmensgruppe mit Spitze in einem Drittstaat ja in den Blick genommen), und mit noch mehr Bauchgrimmen zur Lückenfüllung schreiten, indem er analog dem Unterrichtungsanspruch des nationalen Betriebsrats gegen das verpflichtete Unternehmen Auskunftsansprüche dieses Unternehmen gegenüber den anderen Unternehmen der Gruppe erkennt.

Da war der EuGH viel unbefangener, fragte nicht nach Lücken und stellte einfach fest: Die Richtlinie funktioniere nur richtig, wenn das dem Betriebsrat gegenüber verpflichtete Unternehmen von den anderen Unternehmen unterstützt werde; also habe es entsprechende Unterstützungsansprüche.

Dieses Vorgehen des EuGH beeinflusst das Denken der nationalen Richter. Es verdeutlicht ihnen immer wieder, dass sie das Gemeinschaftsrecht mit den aus dem nationalen Recht vertrauten Auslegungsmethoden nicht richtig erfassen können ${ }^{9}$. Damit werden sie zu Vorlagen an den EuGH ermutigt: Sie können ihn nach einer Auslegung von Gemeinschaftsrecht fragen, die sie bezogen auf nationales Recht möglicherweise nicht in nähere Erwägung gezogen hätten ${ }^{10}$. Und obwohl für die Auslegung innerstaatlichen Rechts die Methodenlehre der jeweiligen Rechtsordnung maßgeblich bleibt ${ }^{11}$, ist es doch nicht auszuschließen, dass unter dem Eindruck der EuGH-Rechtsprechung auch hier teleologische Gesichtspunkte weiter an Bedeutung gewinnen, etwa bei der richtlinienkonformen Auslegung nationaler Vorschriften.

9 Nachdrücklich auch BVerfG v. 9. 1. 2001, NJW 2001, $1267 \mathrm{f}$.

10 Vgl. im Fall Kühne \& Nagel BAG v. 27. 6. 2000, AP EWG-Richtlinie 94/45 Nr. 1.

11 Z.B. EuGH v. 4. 7. 2006, Rs. C-212/04 „Adeneler“, AP Richtlinie 99/70/EG Nr. 1. 


\section{Reibungen}

\section{Anwendung und Durchsetzung des Gemeinschaftsrechts}

Mit diesem Stichwort sind wir bei Fragen der Anwendung und Durchsetzung des Gemeinschaftsrechts angelangt. Diese sind gerade im Arbeitsrecht immer wieder Gegenstand von Urteilen des EuGH. Das liegt daran, dass wir es im geschriebenen Arbeitsrecht der Gemeinschaft fast ausschließlich mit Richtlinien zu tun haben, sieht man einmal von den primärrechtlichen Diskriminierungsverboten wegen der Staatsangehörigkeit (Art. 39 EG) und des Geschlechts (Art. $141 \mathrm{EG)} \mathrm{ab.}$

Nun sind Richtlinien im Verhältnis zwischen privaten Arbeitgebern und deren Arbeitnehmern nicht unmittelbar anwendbar; ihre Inhalte werden vielmehr erst dann für das Arbeitsverhältnis maßgeblich, wenn sie vom nationalen Recht aufgenommen sind. Das ist zwar nicht unumstritten: Der EuGH wird immer wieder gedrängt, Richtlinien unmittelbare Wirkung zwischen Privaten zuzuerkennen. Er ist diesen Vorschlägen, mit denen die Kompetenzordnung der EG umgestürzt würde, aber zu Recht nicht gefolgt ${ }^{12}$ (die insoweit gegenüber öffentlichen Arbeitgebern geltenden Besonderheiten ${ }^{13}$ lasse ich außer Betracht).

Als wichtigstes Instrument zur Durchsetzung von EG-Arbeitsrecht erweist sich die Pflicht zur richtlinienkonformen Auslegung des deutschen Rechts. Hier fühlen wir uns heimisch, ist uns doch das Gebot der verfassungskonformen Auslegung einfachen Rechts schon lange vertraut. Letztere ist allerdings negativ definiert: Sie schließt von mehreren möglichen Auslegungen des einfachen Rechts diejenigen aus, nach denen es gegen Gewährleistungen des Grundgesetzes verstoßen würde. Die Pflicht zur richtlinienkonformen Auslegung hat dagegen positiven Gehalt und ist daher anspruchsvoller: Das nationale Recht ist so auszulegen, dass es das Regelungsziel der Richtlinie erreicht. Insoweit achtet der EuGH aber sorgfältig auf die Balance im Zusammenspiel zwischen Gemeinschaftsrecht und nationalem Recht, das in der Richtlinie angelegt ist: Da Gegenstand der richtlinienkonformen Auslegung das nationale Recht ist, bleibt auch insoweit dessen Methodenkanon maßgeblich. Eine danach unzulässige Auslegung contra legem wird dem innerstaatlichen Richter nicht angesonnen ${ }^{14}$. Schlimmstenfalls muss er auch richtlinienwidriges nationales Recht anwenden ${ }^{15}$.

Dieser einfachen Feststellung muss ich gleich ein „Aber“ hinterher schicken, das die Dinge kompliziert. In letzter Zeit hat nämlich in der arbeitsrechtlichen Judikatur des

12 S. EuGH v. 14. 7. 1994, Rs. C-91/92 „Faccini Dori“, Slg. 1994, I-3347, 3355 f.; v. 5. 10. 2004, Rs. C-397/01 „Pfeiffer“, AP EWG-Richtlinie 93/104 Nr. 12; 4. 7. 2006 (Fn. 11); ebenso z.B. BAG v. 18. 2. 2003, AP BGB $\S 611$ Arbeitsbereitschaft Nr. 12.

13 Z.B. EuGH v. 4. 10. 2001, Rs. C-438/99 „Jiménez Melgar“, AP EWG-Richtlinie 92/85 Nr. 3.

14 EuGH v. 5. 10. 2004 (Fn. 12); v. 4. 7. 2006 (Fn. 11).

15 Z.B. BAG v. 18. 2. 2003 (Fn. 12). 
EuGH eine weitere Rechtsquelle an Bedeutung gewonnen: Das sind die ungeschriebenen Grundsätze des Gemeinschaftsrechts, die primärrechtliche Qualität haben. In der Natur von Ungeschriebenem liegt es, dass es besonders dunkel erscheint und den einen an die Wundertüte denken lässt, den anderen an die Büchse der Pandora. Besonders ein Urteil hat hierzulande für einiges Rätselraten gesorgt, der vieldiskutierte Fall „Mangold"16: Es ging u.a. darum, ob ein deutsches Arbeitsgericht die Befristung eines Arbeitsverhältnisses als wirksam behandeln darf, wenn diese nicht auf einen - sonst erforderlichen - sachlichen Grund gestützt ist, sondern allein darauf, dass der Arbeitnehmer mindestens 52 Jahre alt ist. Das deutsche Recht ( 14 Abs. 3 TzBfG) ließ das damals zu. Damit verstieß es, wie der EuGH ausführlich darlegt, gegen das Verbot der Altersdiskriminierung in der Richtlinie 2000/78/EG. Nach dieser Feststellung wandte sich der Gerichtshof allerdings nicht der Frage des vorlegenden Gerichts nach der Anwendbarkeit richtlinienwidrigen Rechts zu, sondern einer höheren Normenebene: Er erkannte, dass das Verbot der Altersdiskriminierung seinen Ursprung in den gemeinsamen Verfassungstraditionen der Mitgliedstaaten habe und daher ein allgemeiner Grundsatz des Gemeinschaftsrechts sei. Abschließend stellte er fest, dass entgegenstehendes nationales Recht unangewendet bleiben müsse, wobei er wieder auf die Unvereinbarkeit mit der Richtlinie verwies.

Die Konfusion bei uns war groß: Was ist das für ein allgemeiner Grundsatz, den man - jedenfalls in seiner Ausformung durch die Richtlinie 2000/78/EG - bisher in der Verfassung nicht so recht wahrgenommen hatte? ${ }^{17}$ Oder hatte uns der EuGH jetzt doch aufgeben wollen, richtlinienwidriges nationales Recht generell unangewendet zu lassen? Oder meinte er das nur für den Fall eines Verstoßes gegen das „Frustrationsverbot“, der uns seit dem Urteil „Inter-Environnement Wallonie“ 18 vertraut ist? Für eine Erörterung dieser Fragen ist hier kein Raum. Sie verdeutlichen aber ein Anliegen, mit dem ich zum letzten Abschnitt meiner Bemerkungen gelange, der Zusammenarbeit des Bundesarbeitsgerichts mit dem Europäischen Gerichtshof.

\section{Kooperation des $B A G$ mit dem EuGH}

Wie der EuGH immer wieder zu Recht betont, entscheidet er im Vorabentscheidungsverfahren nicht über den Ausgangsrechtsstreit ${ }^{19}$; das bleibt Sache der nationalen Gerichte. Der Gerichtshof gibt vielmehr mit hoher Autorität Auskunft über den Inhalt von Gemeinschaftsrecht. Das scheint mir ein gewisses Maß an Klarheit auch über den Einzelfall hinaus zu gebieten. Für das Urteil über die Klage von Herrn Mangold mag es

16 EuGH v. 22. 11. 2005, Rs. C-144/04, AP Richtlinie 2000/78/EG Nr. 1.

17 Bemerkenswert kritisch hierzu Schlussanträge von GA Mazák vom 15. 2. 2007 in Rs. C-411/05 „Palacios de la Villa“.

18 EuGH v. 18. 12. 1997, Rs. C-129/96, Slg. 1997, I-7411,7449.

19 Z.B. EuGH v. 31. 5. 1995, Rs. C-400/93 „Royal Copenhagen“, AP EWG-Vertrag Art. 119 Nr. 68. 
dem Arbeitsgericht gereicht haben zu erfahren, dass es die Vorschrift über die Zulässigkeit der sachgrundlosen Befristung von Arbeitsverhältnissen mit Arbeitnehmern mit 52 Jahren unangewendet zu lassen hat. Aber die Frage, ob sich das aus der vorrangigen Anwendung eines ungeschriebenen Grundsatzes des Primärrechts, aus dem Frustrationsverbot des Art. 10 EG oder aus einem Verbot der Anwendung richtlinienwidrigen nationalen Rechts ergibt, kann nicht offen bleiben. Wegen der unterschiedlichen Reichweite dieser Regeln ist nicht einmal auszuschließen, dass sie schon im Ausgangsverfahren Bedeutung erlangt. Jedenfalls ist diese Frage, da sie die Grundlagen der Anwendung von Gemeinschaftsrecht betrifft, für eine Vielzahl anderer Verfahren entscheidungserheblich.

Ich möchte nicht missverstanden werden: Dies ist kein Plädoyer dafür, dass der EuGH über die ihm vorgelegten Fragen hinaus Kommentierungen zum Gemeinschaftsrecht liefern soll. Betätigt er sich aber auf einem Terrain, das wir nach seiner bisherigen Rechtsprechung für kartographisch erfasst halten dürfen - im Fall Mangold: die Behandlung richtlinienwidrigen Rechts -, dann sollten die nationalen Richter darauf vertrauen dürfen, dass der Gerichtshof auf Kurskorrekturen ausdrücklich hinweist und bereits Geklärtes nicht in Frage stellt, ohne die nunmehr angestrebte Lösung aufzuzeigen. Auch könnte es trotz der verständlichen Scheu des EuGH vor vertiefter dogmatischer Fundierung seiner Urteile bei der Heranziehung ungeschriebener Rechtsgrundsätze hilfreich sein, Wurzeln und Reichweite des jeweiligen Grundsatzes wenigstens so weit zu umschreiben, dass die innerstaatlichen Gerichte erkennen können, wann sie über seine Anwendung nachzudenken haben.

Schließlich haben die Urteile des Gerichtshofs weit über das Ausgangsverfahren hinaus nicht nur große faktische Bedeutung, wie sie uns auch aus der Rechtsprechung von Revisionsgerichten vertraut ist. Auch rechtlich wirken sie über dieses Verfahren hinaus, denn die vom EuGH erteilten Auskünfte begrenzen, indem sie klärend wirken, die Vorlagepflicht der letztinstanzlichen innerstaatlichen Gerichte. Wie hoch das einzuschätzen ist, erhellt der Blick in Urteile des BAG, deren Begründungen sich mit zunehmender Häufigkeit auch auf Entscheidungen des EuGH stützen. Darüber hinaus kann die Feststellung eines - wie im Fall Mangold bisher unvermuteten - ungeschriebenen Rechtsgrundsatzes eine Vorlagepflicht erst sichtbar machen.

\section{Conclusio}

In der Summe lässt sich allerdings feststellen, dass die Kooperation der deutschen Arbeitsgerichtsbarkeit mit dem EuGH gut funktioniert und auch auf deutscher Seite vom Bestreben getragen ist, dem Gemeinschaftsrecht zur Wirksamkeit zu verhelfen. Natürlich folgt das BAG dem EuGH. Wenn dies im Ausnahmefall erst nach zweifelnder 
Nachfrage und erneuter, konkretisierender Auskunft des EuGH geschieht ${ }^{20}$, so ist das nur Ausdruck der im Verhältnis zwischen dem innerstaatlichen und dem Gemeinschaftsrecht angelegten Spannungen.

20 Instruktiv der Ablauf im Fall Paletta: EuGH v. 3. 6. 1992 Rs. C-45/90 „Paletta I“, AP EWGVerordnung 574/72 Art. 18 Nr. 1; BAG v. 27. 4. 1994, AP LohnFG § 1 Nr. 100; EuGH v. 2. 5. 1996, Rs. C-206/94 „Paletta II“, AP EWG-Verordnung 574/72 Art. 18 Nr. 2; BAG v. 19. 2. 1997 AP, EWG-Verordnung 574/72 Art. 18 Nr. 3. 


\title{
Habent sua fata libelli (= Decisiones)
}

\author{
Marko ILE Ǐ $I \check{C}$
}

\section{Audiatur et altera pars}

The case-law of the European Court of Justice (hereinafter the "ECJ") undoubtedly has a significant influence on the judicial (as well as legislative) activity of member states. Its decisions are perceived with different feelings and cause different reactions. By reactions in this context, I do not mean those of the parties who are directly concerned by a judgment, i.e. member states, European institutions, or the persons who are involved either directly in the procedure before the ECJ or indirectly through a national judicial procedure where the national court refers a preliminary ruling to the ECJ. Indeed, their reactions are also interesting; typical, above all, are the reactions to procedures against member states who are obliged to respect decisions on the basis of Article 10 of the EC Treaty.

In this article, I would like to mention some opinions expressed by the academic community in the course of reactions to decisions of the ECJ. Taking into account that I have been kindly invited to comment on some questions about our case-law in the area of labour law, I would like to take this opportunity to limit my presentation to the caselaw which has recently attracted significant attention in terms of theory and which will be a "continued" in the case-law.

Probably you have already guessed that I will speak about the challenges which have been posed by the judgment of the ECJ in the Mangold case, ${ }^{1}$ which has already been mentioned today. It goes without saying that I can only comment on my own behalf and not on that of the ECJ. And the fact that I was a member of the chamber which decided the case in no way means that I would reveal anything that could violate the judge's oath of confidentiality.

Do not get me wrong, I am not commenting on this judgment because I am offended. Quite the opposite is true: the judgment is a confirmation of the statement that the ECJ is an important Impulsgeber (impulse giver) (also) in the area of labour law. And finally, such a vivid response in the literature ${ }^{2}$ is sufficient proof of this.

1 C-144/05, 22.11.2005.

2 My research has found more than 50 published articles. Only in the small country of Slovenia two articles on this case were recently published. Korpič Horvat: Diskriminacija pri zaposlovanju zaradi starosti, in. Delavci in delodajalci, 2-3/2007, p. 313-328; Kresal: Prepoved diskriminacije na področju dela v luči prakse Sodišča ES, in: Prepoved diskriminacije, Ljubljana 2007, p. 45-66. 